\title{
Adaptive ECG Biometric Recognition: a Study on Re-Enrollment Methods for QRS Signals
}

\author{
Ruggero Donida Labati, Vincenzo Piuri, Roberto Sassi, Fabio Scotti, Gianluca Sforza \\ Department of Computer Science \\ Università degli Studi di Milano \\ Milano, 20122, Italy. \\ \{ruggero.donida, vincenzo.piuri, roberto.sassi, fabio.scotti, gianluca.sforza\}@ unimi.it
}

\begin{abstract}
The diffusion of wearable and mobile devices for the acquisition and analysis of cardiac signals drastically increased the possible applicative scenarios of biometric systems based on electrocardiography (ECG). Moreover, such devices allow for comfortable and unconstrained acquisitions of ECG signals for relevant time spans of tens of hours, thus making these physiological signals particularly attractive biometric traits for continuous authentication applications. In this context, recent studies showed that the QRS complex is the most stable component of the ECG signal, but the accuracy of the authentication degrades over time, due to significant variations in the patterns for each individual. Adaptive techniques for automatic template update can therefore become enabling technologies for continuous authentication systems based on ECG characteristics.

In this work, we propose an approach for unsupervised periodical re-enrollment for continuous authentication, based on ECG signals. During the enrollment phase, a "super" template obtained from a fixed number of samples is stored in the gallery. In continuous authentication, an update condition is periodically verified. If the condition is satisfied, confirming that the fresh data pertain to the stored identity, an update strategy is applied to fuse the fresh data with the "super" template. Different update conditions and update strategies are presented and evaluated.

Tests have been performed on a significantly large public dataset of $24 \mathrm{~h}$ Holter signals acquired in uncontrolled conditions, proving that the proposed approach obtains a relevant accuracy, which increases with respect to traditional biometric approaches based on a single enrolled template for each individual.
\end{abstract}

Index Terms-Biometrics, ECG, Continuous Authentication, Re-enrollment, Adaptive Biometrics.

\section{INTRODUCTION}

Recent studies in biometrics focus on the use of onedimensional physiological signals, e.g., electrocardiograms (ECG), electroencephalograms (EEG) [1], phonocardiograms (PCG) [2], and photoplethysmograms (PPG) [3], [4].

Physiological signals present some important advantages [5]:

- they are more difficult to counterfeit with respect to traits like face, fingerprint or voice.

- the analysis of physiological signals can reveal information related to psychological states, as well as physiological and clinical conditions of the person.

- they can usually be acquired for long periods of time without requiring explicit efforts from the user, making them particularly suitable for continuous authentication applications.
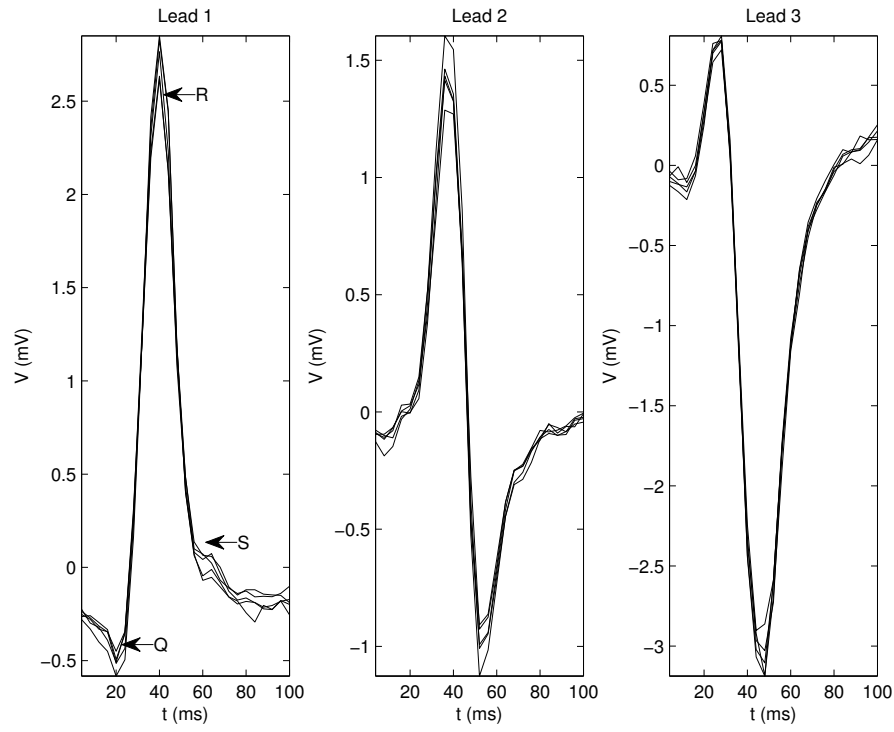

Fig. 1. The QRS complex. Five aligned and superimposed heart beats of an individual measured at three different leads.

ECG-based biometric systems are of particular relevance, given the fact that ECG is one of the most widespread and mature technologies in clinical analyses. Previous studies on ECG-based biometric systems reveal that ECG signals have sufficient discriminability so that accurate results can be obtained [6]. However, ECG-based systems have to overcome different practical issues due to the leads placement, scalability, heart rate variability, and, most notably, the time-varying nature (permanence issue) of the ECG signals [5]. In fact, a performance decrease of the system can usually be observed when samples are captured with sufficient distance of time and in uncontrolled conditions [5].

Research proved that the QRS complex is that part of the ECG which is more robust to temporal variations [7]. It represents the depolarization of the right and left ventricles. An example of this waveform, for the three different leads of an ECG signal, is shown in Fig. 1.

In our previous work [8], we presented a preliminary study on continuous authentication systems based on ECG signals. The main goal was to analyze the permanence of the QRS complex. The persistency analysis showed that the recognition 
performance is strictly related to the time elapsed between the acquisition of the signals under comparison. In particular, we found that the persistence of the QRS complex in the entire record is limited to 2 hours out of 24 . This result certainly depends on the peculiarities of the ECG signal pointed out above, such as the variability of the signal in time, which impacts on the recognition performances.

During the enrollment phase, features extracted from biometric data are associated ("labelled") to the corresponding user's identity. However, variations between the enrolled biometric traits (labelled) and the ones obtained after the enrollment stage (unlabelled), constitutes a significant issue for biometric systems designed for continuous recognition, whose performance can degrade quickly. With the aim of increasing the classification results, adaptive techniques may be considered, as suggested in [9]. In these systems, new biometric data collected over the time, if correctly labelled, can be exploited to adapt the system to input data variations.

In real operational scenarios, there are situations where variations of data acquired by biometric systems can be of considerable importance [10]. That happens for example with changes of the environmental conditions (e.g., illumination), or variations in the interaction of the user with the sensor (e.g., pose of the face, start of a new activity), or even aging of the biometric trait, etc. All these cases show a large intraclass variability of the biometric pattern, a condition that can lead to templates with reduced capacity of representing the enrolled data, and in turn to reduced recognition performance.

Temporal variations in the biometric patterns can be caused by the non-stationary nature of the source of the data. This is particularly true with ECG, where autonomic modulations, respiration and postural changes depend on the activities done by the person, and the pattern can vary during the day. Data recordings in non-stationary cases can be reasonably exploited in order to implement adaptive systems that can improve performances while they are operating. Holter ECG recordings could help for tackling the intrinsic variability of this trait, considering that a lot of new biometric data are made available over 24 hours.

There are cases in which, in a single enrollment session, multiple templates can be acquired. For example, changes in the pose of the face can be acquired in the enrollment session, asking the collaboration of the user. Furthermore, enrollment sessions repeated more times surely help to capture part of the temporal variations of biometric traits of an individual. However, frequent re-enrollment sessions can be expensive, in terms of effort of the user, storage capability, length of the session, etc. By contrast, ECG data may be recorded over 24 hours without having a significant impact on the normal activities of the user.

Taking ground from the conclusions of our previous work [8], we propose a novel biometric recognition approach for continuous authentication, based on ECG signals. Exploiting continuous ECG recordings, information coming from variations over time in the ECG signal can be captured and used to adapt the template enrolled previously. The main goal of this work is to analyze different strategies for template's updating, in order to improve the accuracy of the recognition method.

The contribution of the paper is twofold. First, for the first time in the literature, different re-enrolment techniques are applied to long-term ECG signals and compared by using a publicly available dataset. Second, a specific set of adaptive methods are proposed in order to exploit the peculiarities of the QRS complex, with respect to more accurate hard biometric traits.

The paper is structured as follows. Section II describes the state of the art related to ECG biometric recognition and adaptive biometric systems. The proposed ECG adaptive recognition approach is then presented in Section III. The results we obtained are discussed in Section IV. Finally, Section $\mathrm{V}$ proposes conclusions and final remarks.

\section{RELATED WORKS}

Most of the studies on ECG biometric recognition are based on single lead signals [5]. However, researches which use multiple leads have also been presented [11], [12], since the increased quantity of information allows for improvements in the accuracy and reduces the effects of the noise on recognition. Several techniques in the literature extract information from all the components of the ECG [13]. However, methods considering only the QRS component, as the most stable-intime part of the ECG, have also been suggested for biometric recognition: [14] is based on functional approximation of the signal, and [15], [16], [17] use computational intelligence and statistical techniques in the matching strategy.

In this work, we chose an approach based only on the QRS complex, obtained from multiple leads, for continuous authentication. To the best of our knowledge, in the literature there are only a few studies tackling continuous authentication based on ECG signals [18], [19], [20]. The method presented in [18] performs authentications every 35 heartbeats, whereas in [19], [20] time intervals of $5 \mathrm{~s}$ are used. These methods, however, have been evaluated on small datasets of signals encompassing a maximum period of 15 minutes. Given this relevant limit, the real applicability of the proposed techniques on long-term ECG recognition is not proven.

Continuous authentication systems based on more commonly used biometric traits have also been studied [21]. Face characteristics are used in [22] for continuous recognition of users working in front of a computer. Multimodal approaches have also been proposed, in order to obtain better accuracy results. The system described in [23] is designed for the same purpose (i.e. computer user authentication), fusing soft biometric features - as primary elements of the matchingwith face features and password. The system in [24] is based on both fingerprint and face features, and uses a holistic strategy to fuse the matching scores. A multimodal approach based on fuzzy systems is proposed in [25], where face and fingerprint data are used together for continuous authentication applications. 
Most of these systems perform the enrollment once. Novel studies instead propose a continuous update of the stored templates [23], and there are also examples of strategies for periodically updated biometric databases [26], [27].

In [9], the authors consider adaptive biometric systems as systems performing a semi-supervised learning task, using initially labelled data and then unlabelled training examples. Unlabelled archives of data are acquired when the system operates, while labelled data are collected during an initial enrollment phase. Some systems have been developed using semi-supervised learning methods, such as [28], [29]. Using a similar approach, unlabelled archives were exploited in recognition systems to adapt fingerprint [30], [31] or of face [32] templates.

\section{A. Adaptive Fingerprint Recognition}

Regarding the recognition of fingerprints, the techniques proposed in the literature to address a large intraclass variability aim at the creation of a gallery of representative templates, or, from multiple samples, at producing a single "super" template [9]. In [10] authors proposed two methods to select a gallery of templates representative of multiple fingerprints. The methods adopt respectively a clustering strategy and a maximum similarity criteria. Methods which generate super templates by merging multiple fingerprints are used by [30], [31]. They proposed the merge of minutiae points of different fingerprints into a single minutia. All these methods process fingerprints on-line, one by one, and update the template accordingly, meaning that the sequence of input is crucial. Moreover, only fingerprints recognized as genuine with high reliability are used for the update.

\section{B. Adaptive Face Recognition}

Many systems were developed for adaptive face recognition. An incremental update of the galleries of face images was proposed by Okada and von der Malsburg, who described a prototype system for face recognition in video streams [33]. Only views recognized with high reliability in the input video are added to the galleries. On the contrary, when the identity of the face image is unknown, a new entry is created in the gallery. Another early work in this area is [34], where the authors proposed a self-organizing approach to face recognition in video, also to address the "forgetting" issue (that is, how the system may fail to "remember" outdated data) and save memory. The work [35] presented "Argus", an adaptive system for automatic face identification of visitors entering a building. However the system was not self-adaptive as it was supervised by the watchmen during its operation, who assigned an identity to unknown visitors' images. Incremental update is used also in a face recognition system based on PCA [32]. More recently, Nagy introduced a new concept called CAVIAR (Computer Assisted Visual Interactive Recognition) aiming to exploit the different abilities of humans and computers in visual recognition [36], [37]. The user provides feedbacks to the CAVIAR-based system, which may be used to adapt the recognition models.

\section{Adaptive ECG Recognition}

At the present, we have knowledge of very few works on adaptive approaches applied to ECG. Home telemonitoring is the application setting in [20], where a biometric system is designed to identify users from ECG signals for the sake of their security. The authors claim that the system is robust in user identification, and capable of adapting to physical or emotional variations of the person monitored. However, the adaptation criterion does not consider the possible presence of impostors after enrollment, as it might happen in a real authentication scenario.

The approach presented here does not include a standard technique taken from the literature "as it is", since these techniques refer to the re-enrollment of biometric traits, like fingerprints, characterized by higher accuracy with respect to the ECG. On the contrary, we tested different methods which modify the standard approach to adaptive recognition, and make it suitable to the essential characteristics of ECG data (e.g., the strong variability).

\section{The Proposed APPROACH}

In this paper, we propose a novel adaptive approach for continuous biometric recognition based on the analysis of ECG signals collected in uncontrolled scenarios from portable devices (Holter acquisitions). The approach exploits the high temporal variability of ECG signals by adaptively modifying the knowledge base of the biometric system in an unsupervised manner [26]. The main idea of the approach consists in automatically updating the gallery of biometric templates by analyzing the results obtained from biometric queries.

Differently from most of the adaptive biometric systems in the literature, which are designed to reduce the intraclass variability of the gallery or to update the knowledge base of the system considering slow aging processes in long periods of time, the proposed approach considers quick and unpredictable variations of the ECG signal due to differences in activities performed by the user or, possibly, emotional states. The base of the approach consists in the biometric recognition method previously presented in [8].

The proposed adaptive template update strategy is general and can also be applied to different biometric recognition systems. It can be considered as a tailoring of the techniques described in [30], [38], [31] to biometric data undergoing quick variations, like ECG signals. The schema of the proposed approach is shown in Fig. 2.

\section{A. Feature Extraction and Matching}

As proposed in [8], in this work we use multiple leads in order to obtain accurate recognitions, and we consider samples with fixed time duration of $\Delta_{t}$ seconds. The biometric recognition method can be divided into the following steps:

1) noise reduction

2) segmentation;

3) feature extraction;

4) matching;

5) score fusion. 


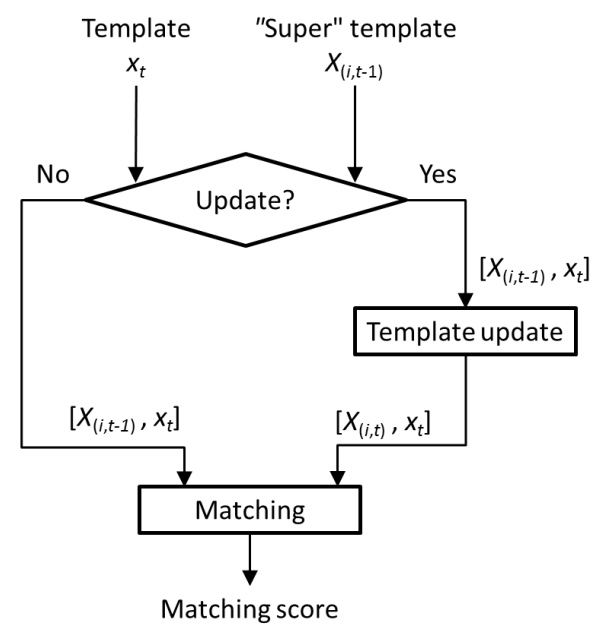

Fig. 2. Schema of the proposed template update approach.

The noise reduction step is performed by using a notch IIR filter to remove the noise introduced by the $50 \mathrm{~Hz}$ power transmission lines and then by applying a third order high-pass Butterworth filter with cutoff frequency of $0.5 \mathrm{~Hz}$ to normalize the signal's baseline.

The segmentation algorithm employed first estimates a list of $\mathrm{R}$ fiducial points (the main positive peak in the QRS complex) in the ECG signal by using an automatic labeling software (Vision Premier, SpaceLab-Burdick Inc.). For each point R, the corresponding QRS complex is then estimated by using a fixed time window centered in $\mathrm{R}$ (from $-50 \mathrm{~ms}$ to $+70 \mathrm{~ms}$ ). The result of this step is a vector $H$ of QRS signals.

In the next step, an iterative algorithm creates a feature vector composed by a maximum of $m$ QRS signals with the best correlations with the average QRS pattern of $H$.

The matching process consists in searching for the maximum correlation value between the corresponding QRS complexes of two feature vectors. We consider a template $x$ as a set of heartbeat signals obtained from the different leads. In order to obtain higher accuracy, the matching scores obtained from signals of different leads are finally fused using well-known algorithms. Differently from the work presented in [8], as matching function we compute the median of the matching scores of the single leads, since it improves the update strategies with more accuracy on datasets composed by higher numbers of samples (a detailed description of this results is given in the experimental section).

\section{B. Online Template Update}

During the enrollment phase, the proposed method creates a "super" template $X$ composed by $N$ templates $x_{j}$, with $j \in$ $[1: N]$. The "super" template $X$ is then updated online during the execution of the continuous verification system.

Since every template $x$ is computed from samples representing ECG signals with a fixed duration of $\Delta_{t}$ seconds, the enrollment phase requires a total of $N \times \Delta_{t}$ seconds. Therefore, the value $N$ should be chosen as a tradeoff between accuracy and usability. In this work, we considered a maximum enrollment time of 15 minutes, which is comparable to the time usually employed by medical doctors to check the status of the Holter apparatus when applied to patients.

During the continuous verification process, for each identity comparison, the proposed approach first verifies if it is possible to update a stored "super" template $X$. If the update condition is reached, template update algorithms are then applied to $X$. Finally, the matching score $s$ is computed.

1) Update condition: Considering an identity verification performed at the instant of time $t$, the fresh template $x_{t}$ is considered as pertaining to the individual $i$ only when the following condition is satisfied:

$$
\text { Update }=\left\{\begin{array}{l}
1 \text { if } \max _{j=1 \ldots N}\left[\operatorname{match}\left(X(j)_{(i, t-1)}, x_{t}\right)\right]>t_{u}, \\
0 \text { otherwise }
\end{array},\right.
$$

where $X(j)_{(i, t-1)}$ are the templates composing the "super" template $X_{(i, t-1)}$ associated to the identity $i$ at the instant of time $t-1, \operatorname{match}(\cdot)$ is the matching function, and $t_{u}$ is an empirically estimated threshold.

In order to avoid identity substitutions in the biometric database, the threshold value $t_{u}$ should be sufficiently strict to avoid all impostors to access the system. For this reason, we suggest to set the value of $t_{u}$ to the threshold value corresponding to Zero False Match Rate (ZeroFMR) of the initial gallery, or to estimate this value from other available databases acquired in similar conditions. Nevertheless, in scenarios with very noisy and variable biometric acquisitions, this threshold could be also set to different values, according to the security/applicability (FAR/FRR) tradeoff of the applications.

2) Template update: Since ECG signals can present both changes due to physiological conditions and noise inserted by environmental factors, we investigate different algorithms for template updating. The goals are different: to maximize the intraclass variability covered by templates pertaining to the "super" templates in the gallery (reducing the effects of the physiological variability of the signal), or to minimize the intraclass variability of the templates pertaining to the "super" templates in the gallery (reducing the effects of external noise). The implemented algorithms are described in the following:

- Update A - The "super" template $X_{(i, t)}$ is obtained by substituting the template $X(k)_{(i, t-1)}$ from $X_{(i, t-1)}$ with $x_{t}$, where:

$$
k=\underset{j=1 \ldots N}{\operatorname{argmax}}\left\{\operatorname{match}\left[X(j)_{(i, t-1)}, x_{t}\right]\right\} .
$$

- Update B - The "super" template $X_{(i, t)}$ is obtained similarly to Update A, but computing $k$ as follows:

$$
k=\underset{j=1 \ldots N}{\operatorname{argmin}}\left\{\operatorname{match}\left[X(j)_{(i, t-1)}, x_{t}\right]\right\} .
$$

- Update C - The templates pertaining to the "super" template $X_{(i, t-1)}$ and the probe template $x_{t}$ are stored in a vector $V$.

All the possible $(N+1) \times(N+1)$ matching scores between the elements of $V$ are then computed and stored in the matrix $Y$. A similarity weight is then computed 
for every template $j$ of $V$ by estimating the median of every column of $Y$ as follows:

$$
W(j)=\operatorname{median}_{k=1 \ldots N+1}[Y(j, k)] .
$$

The template $V\left(n_{r}\right)$ is then removed from $V$ and the "super" template $X_{(i, t)}$ is finally set as equal to $V$. The template number $n_{r}$ is selected as follows:

$$
n_{r}=\underset{j=1 \ldots N+1}{\operatorname{argmin}}[W(j)] .
$$

- Update D - The "super" template $X_{(i, t)}$ is obtained similarly to Update $\mathrm{C}$, but computing $n_{r}$ as follows:

$$
n_{r}=\underset{j=1 \ldots N+1}{\operatorname{argmax}}[W(j)] .
$$

3) Matching score computation: The matching score $m_{s}$ is finally computed as follows:

$$
m_{s}=\max _{j=1 \ldots N}\left\{\operatorname{match}\left[X(j)_{(i, t)}, x_{t}\right]\right\} .
$$

\section{EXPERIMENTAL RESUlts}

Experiments have been performed to evaluate the proposed adaptive template update approach, in continuous authentication scenarios, based on Holter ECG acquisitions. The analyses focused on the evolution of the system's performances in time. In particular, we performed both traditional tests, to estimate the verification accuracy, and tests simulating the operative conditions of a continuous authentication system. For the latter, we assumed that a set of users is continuously authenticated, with periodic checks after $\Delta_{a} \mathrm{~s}$. We assumed the value of $\Delta_{a}$ to be larger or equal than the length of the template employed $\Delta_{t}$.

Using a set of ECG signals with a duration of $T$ seconds, collected from $M$ individuals, the verification performance of the biometric system is usually evaluated by dividing each signal in $S$ samples and then performing $[(M \times S) \times(M \times S-$ 1)] $/ 2$ identity comparisons. In order to simulate a continuous verification system, we performed $M \times S$ identity comparisons for each instant of time $t$. Unlabelled templates at time $t$ are therefore compared only with the templates composing the gallery at time $t$. Thus, the total number of performed identity comparisons is $M \times(M-1) \times S$.

\section{A. The Biometric Dataset}

The database we employed was derived from the E-HOL03-0202-003 (Intercity Digital Electrocardiogram Alliance IDEAL) database [39], which is composed of 202 digital 24-hours Holter recordings (SpaceLab-Burdick Inc.) obtained from 202 individuals. The acquisitions were performed without any restrain or control on the activities performed. Males and females are equally distributed. The ECG signals were acquired with a three pseudo-orthogonal leads configuration ( $\mathrm{X}, \mathrm{Y}$ and $\mathrm{Z}$ ). The sampling frequency is $200 \mathrm{~Hz}$ and the amplitude resolution is $10 \mu \mathrm{V}$.

In 17 recordings, at least one channel was corrupted by artifacts and noise; they were excluded from further analyses. Thus we only considered samples from 185 individuals out of
202. Due to the high computational costs of the tests we designed, we further selected three subsets of the Holter database to validate the proposed approach, which are composed as follows:

- DB 1 - 4,200 samples acquired from 100 individuals. For each individual, 3.5 hours of ECG signals are considered, thus obtaining 42 samples.

- DB 2 - 8,400 samples acquired from 100 individuals. For each individual, 7 hours of ECG signals are considered, thus obtaining 84 samples. Half of the samples pertain also to DB 1. DB 2 was designed to evaluate the continuous authentication method over a time span representing a working day.

- DB 3 - 7,770 samples acquired from 185 individuals. For each individual, 3.5 hours of ECG signals are considered, thus obtaining 42 samples. Samples of 100 individuals pertain also to DB 1. DB 3 was designed to test continuous authentication methods with the larger number of users.

\section{B. Parameters' values employed}

An important parameter of the template update method is the threshold value $t_{u}$. In order to avoid identity substitutions, it must correspond to the ZeroFMR (Zero False Matche Rate) of the biometric system. Nevertheless, in some applicative contexts, it might be reasonable to relax this requirement. For this reason, we performed experiments with different values of the parameter $t_{u}$ :

- $t_{u}=t_{E E R}$, where $t_{E E R}$ is the threshold value corresponding to the Equal Error Rate (EER), as computed on the enrolled templates;

- $t_{u}=t_{Z \text { EroFMR }}$, where $t_{Z e r o F M R}$ is the maximum impostor score computed from the enrolled templates.

A second relevant parameter is the number $N$ of templates composing the "super" templates. In order to limit the enrollment time to 15 minutes, we used samples with a duration $\Delta_{t}=5$ minutes and $N=3$.

\section{Verification Accuracy (baseline estimation)}

As a reference for the successive tests, we report the results obtained evaluating the verification accuracy of the considered biometric recognition system on the three subsets of the full database, listed before. The test involved: 8,817,900 identity comparisons for DB 1, 35,275,800 identity comparisons for DB 2, and 30,182,565 identity comparisons for DB 3. The Receiver Operating Characteristic (ROC) curves are presented in Fig. 3 and the numerical results are summarized in Table I. A comparable accuracy was obtained on DB 1 and DB 3, while performances decreased on DB 2, which is composed by samples acquired over a longer time span. As an example, the EER obtained on DB 1 is equal to $5.68 \%$, while the EER obtained on DB 2 is equal to $6.86 \%$.

\section{Continuous Verification Accuracy}

We evaluated the accuracy of the proposed template update approach in different configurations, by simulating continuous 


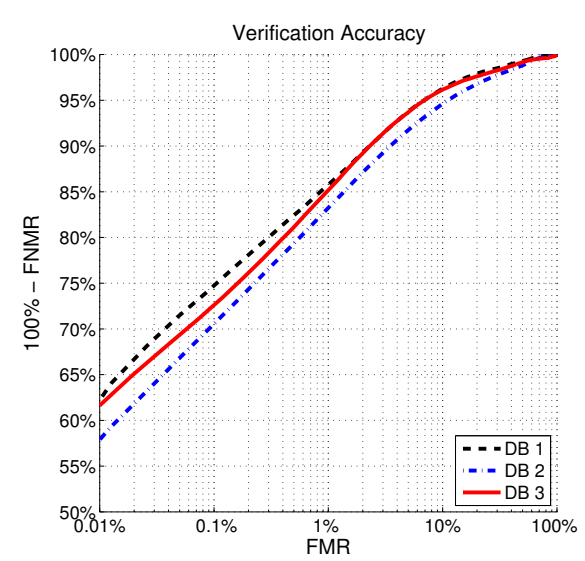

Fig. 3. ROC curves obtained analyzing the system accuracy in verification modality. The obtained EER values are $5.68 \%$ on DB $1,6.86 \%$ on DB 2, and $5.68 \%$ on DB 3 .

TABLE I

VERIFICATION ACCURACY ON THE CONSIDERED DATASETS.

\begin{tabular}{ccc}
\hline \hline Dataset & $\begin{array}{r}\text { EER } \\
(\boldsymbol{\%})\end{array}$ & $\begin{array}{c}\text { FRR (\%) @ } \\
\text { FAR = 1\% }\end{array}$ \\
\hline DB 1 & 5.68 & 14.08 \\
DB 2 & 6.86 & 16.57 \\
DB 3 & 5.68 & 13.73 \\
\hline \hline
\end{tabular}

verification. For these tests, we simulated the continuous authentication in ideal conditions, when no attacks are performed by impostors to the template update module. Since every enrolled "super" template is composed by three templates, the tests involved: 390,000 identity comparisons for DB 1, 810,000 identity comparisons for DB 2, and 1,334,775 identity comparisons for DB 3. We considered two configurations of the system: i) $t_{u}=t_{\text {ZeroFMR }}$, the decision threshold is set to the working point ZeroFNMR of the gallery; and ii) $t_{u}=t_{E E R}$, the decision is set to the working point EER of the gallery. The results are presented in Table II.

The best performances of the method were obtained when using the configuration Update A, for which the highest accuracy on all the datasets was obtained. Moreover, the results show that the choice of the threshold $t_{u}$ drastically influences the performance of the system and when using $t_{u}=t_{E E R}$, the system displayed higher accuracy. However, this configuration of the biometric system is more vulnerable to attacks with respect to $t_{u}=t_{\text {ZeroFMR }}$.

\section{E. Comparison with the Baseline Accuracy Values}

In order to prove the validity of the proposed approach, we compared the performances of the sequent biometric recognition systems:

- Baseline 1 - the system does not use template update strategies and stores one template per individual in the gallery;

- Baseline 2 - the system does not use template update strategies and stores a "super" templates composed by 3 templates;
TABLE II

RESULTS OBTAINED SIMULATING A CONTINUOUS VERIFICATION SCENARIO.

\begin{tabular}{|c|c|c|c|c|c|}
\hline Dataset & $\begin{array}{l}\text { Theshold } \\
\text { Value }\end{array}$ & $\begin{array}{l}\text { Update } \\
\text { Method }\end{array}$ & $\begin{array}{r}\text { EER } \\
(\%)\end{array}$ & $\begin{array}{c}\text { FRR (\%) @ } \\
\text { FAR }=1 \%\end{array}$ & $\begin{array}{c}\% \text { of } \\
\text { Updates }\end{array}$ \\
\hline DB 1 & $t_{E E R}$ & Update A & 1.47 & 1.62 & 98.38 \\
\hline DB 1 & $t_{E E R}$ & Update B & 1.98 & 2.15 & 97.85 \\
\hline DB 1 & $t_{E E R}$ & Update C & 5.21 & 11.92 & 94.33 \\
\hline DB 1 & $t_{E E R}$ & Update D & 2.13 & 2.44 & 97.97 \\
\hline DB 1 & $t_{Z \text { eroF } M R}$ & Update A & 3.89 & 8.18 & 76.46 \\
\hline DB 1 & $t_{Z \text { eroF } M R}$ & Update B & 4.10 & 8.64 & 75.46 \\
\hline DB 1 & $t_{Z \text { eroF } M R}$ & Update C & 4.70 & 10.59 & 67.82 \\
\hline DB 1 & $t_{Z \text { eroF } M R}$ & Update D & 4.24 & 8.56 & 74.36 \\
\hline DB 2 & $t_{E E R}$ & Update A & 1.34 & 1.44 & 98.56 \\
\hline DB 2 & $t_{E E R}$ & Update B & 2.27 & 2.46 & 97.54 \\
\hline DB 2 & $t_{E E R}$ & Update C & 5.64 & 13.11 & 93.10 \\
\hline DB 2 & $t_{E E R}$ & Update D & 2.73 & 4.20 & 97.57 \\
\hline DB 2 & $t_{Z \text { eroF } M R}$ & Update A & 4.22 & 8.14 & 75.73 \\
\hline DB 2 & $t_{Z \text { eroF } M R}$ & Update B & 4.84 & 9.85 & 71.80 \\
\hline DB 2 & $t_{Z \text { eroF } M R}$ & Update C & 5.70 & 12.11 & 60.25 \\
\hline DB 2 & $t_{Z \operatorname{ero}} F M R$ & Update D & 5.03 & 10.27 & 68.98 \\
\hline DB 3 & $t_{E E R}$ & Update A & 1.97 & 2.00 & 98.00 \\
\hline DB 3 & $t_{E E R}$ & Update B & 2.54 & 2.61 & 97.39 \\
\hline DB 3 & $t_{E E R}$ & Update C & 4.79 & 11.16 & 93.76 \\
\hline DB 3 & $t_{E E R}$ & Update D & 2.66 & 3.34 & 97.34 \\
\hline DB 3 & $t_{Z \text { eroF } M R}$ & Update A & 3.83 & 7.66 & 72.38 \\
\hline DB 3 & $t_{Z \text { eroF } M R}$ & Update B & 3.91 & 8.84 & 72.32 \\
\hline DB 3 & $t_{Z \text { eroF } M R}$ & Update C & 4.44 & 10.40 & 63.87 \\
\hline DB 3 & $t_{Z \text { eroFMR }}$ & Update D & 4.05 & 8.54 & 70.76 \\
\hline
\end{tabular}

- Adaptive EER - the system uses the proposed template update approach in the best configuration (Update A), with threshold $t_{u}=t_{E E R}$;

- Adaptive ZeroFMR - the system uses the proposed template update approach in the best configuration (Update A), with threshold $t_{u}=t_{\text {ZeroFMR }}$.

Fig. 4 presents the ROC curves obtained while the numerical results are summarized in Table III. They show that the proposed approach outperforms the baseline methods on the biometric datasets considered. The performance increase is particularly evident on DB 2 (the dataset composed by Holter signals acquired in the longer time period). As an example, the EER decreases from $7.36 \%$ (Baseline 1) to $4.22 \%$, for the configuration with $t_{u}=t_{Z \text { EeroFMR }}$, and to $1.34 \%$, for the configuration with $t_{u}=t_{E E R}$.

To evaluate the effectiveness of the proposed approach, we have also analyzed the evolution of the recognition performances during time. As an example, Fig. 5 presents the EER obtained at each instant of time on DB 3. It is possible to observe that the method effectively reduces the error increase during time, due to the variability of ECG signals.

\section{F. Robustness to Attacks}

In order to evaluate the robustness of the template update strategy, we simulated impostors' attacks to this module. We considered the best configuration of the proposed approach (Update A) with the threshold $t_{u}=t_{\text {ZeroFMR }}$. In the simulations of the continuous authentication scenario (Subsection IV-D), we quantified the number of impostor comparisons with matching score greater than $t_{u}$. In fact, the threshold value $t_{Z \text { ZeroFMR }}$ was computed only on the templates enrolled and not on the full set of templates. The results we obtained are reported in Table IV. The estimated percentages of successful 
(a)

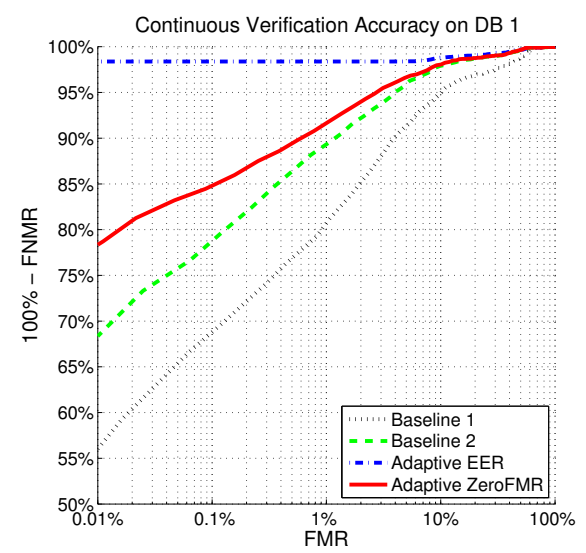

(b)

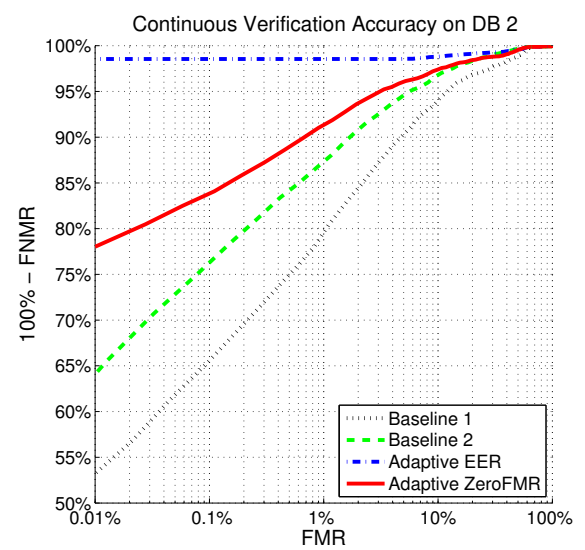

(c)

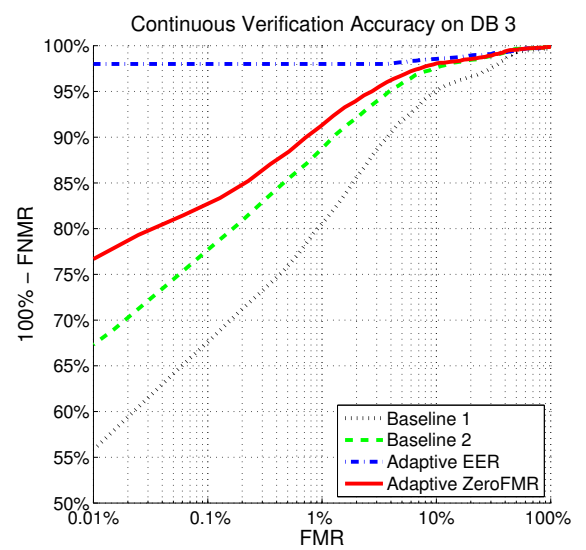

Fig. 4. ROC curves obtained by applying the baseline algorithms and proposed approach in the best configuration (Update A) and different values of the threshold $t_{u}$ on: (a) DB 1, (b) DB 2, and (c) DB 3. It is possible to observe that the proposed method obtains higher accuracy with respect to the baseline algorithms.

TABLE III

COMPARISON BETWEEN THE PROPOSED APPROACH AND THE BASELINE METHODS.

\begin{tabular}{clcc}
\hline \hline Dataset & Method & $\begin{array}{r}\text { EER } \\
(\boldsymbol{\%})\end{array}$ & $\begin{array}{c}\text { FNMR (\%) @ } \\
\text { FMR = 1\% }\end{array}$ \\
\hline DB 1 & Baseline 1 & 6.80 & 18.80 \\
DB 1 & Baseline 2 & 4.49 & 10.62 \\
DB 1 & Adaptive EER & 1.47 & 1.62 \\
DB 1 & Adaptive ZeroFMR & 3.89 & 8.18 \\
\hline DB 2 & Baseline 1 & 7.36 & 19.61 \\
DB 2 & Baseline 2 & 5.23 & 11.95 \\
DB 2 & Adaptive EER & 1.34 & 1.44 \\
DB 2 & Adaptive ZeroFMR & 4.22 & 8.14 \\
\hline DB 3 & Baseline 1 & 6.71 & 18.93 \\
DB 3 & Baseline 2 & 4.40 & 11.23 \\
DB 3 & Adaptive EER & 1.97 & 2.00 \\
DB 3 & Adaptive ZeroFMR & 3.83 & 7.66 \\
\hline \hline
\end{tabular}

attacks on the datasets can be considered as satisfactory for most of the biometric recognition applications based on ECG signals.

\section{CONCLusion}

The paper presented a study focused on re-enrollment adaptive methods in ECG biometric recognition, with specific reference to the QRS complex. It introduced the design and test of different methods meant to create and adapt in time a set of QRS templates (a "super" template), associated to each user, by efficiently managing the variability of the ECG signals. Different strategies to update the "super' template are presented and compared with respect to a normal recognition scenario without re-enrollment. The work considered a dataset with a relevant time span and with a significant number of users with respect to previously published studies. Moreover, the study confirmed the differences with respect to similar techniques used in hard biometric templates, such as fingerprints. Results are encouraging, and the proposed study shows that is possible to tune the parameters of the methods in order to effectively select the security level of the application. (a)

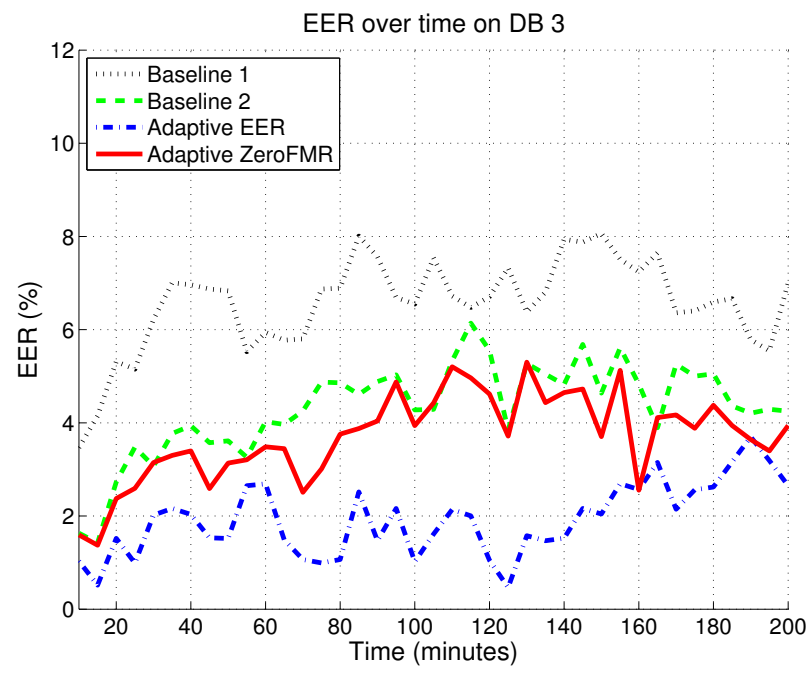

Fig. 5. Evolving of the EER during time obtained by applying the baseline algorithms and proposed approach in the best configuration (Update A) and different values of the threshold $t_{u}$ on DB 3 . It is possible to observe that proposed adaptive method effectively reduce the error increasing during time.

\section{ACKNOWLEDGMENT}

This work was partly funded by the European Commission under the project ABC4EU (contract n. FP7-312797) and the Italian Ministry of Research within PRIN project "GenData 2020" (2010RTFWBH).

Data used for this research were provided by the Telemetric and Holter ECG Warehouse of the University of Rochester (THEW), NY.

\section{REFERENCES}

[1] W. Khalifa, A. Salem, M. Roushdy, and K. Revett, "A survey of EEG based user authentication schemes," in Proc. of the 8th Int. Conf. on Informatics and Systems, 2012, pp. 55-60.

[2] F. Beritelli and S. Serrano, "Biometric identification based on frequency analysis of cardiac sounds," IEEE Trans. on Information Forensics and Security, vol. 2, pp. 596-604, 2007. 
TABLE IV

ROBUSTNESS TO ATTACKS.

\begin{tabular}{c|c|c}
\hline \hline & \multicolumn{2}{|c}{ Matching scores $>t_{u}$} \\
Dataset & Number & $\boldsymbol{\%}$ \\
\hline DB 1 & 30 & 0.0077 \\
DB 2 & 38 & 0.0047 \\
DB 3 & 37 & 0.0028 \\
\hline \hline
\end{tabular}

[3] Y. Y. Gu, Y. Zhang, and Y. Zhang, "A novel biometric approach in human verification by photoplethysmographic signals," in Proc. of the 4th IEEE Annual Int. Conf. of the Engineering in Medicine and Biology Society (EMBS), 2003, pp. 13-14.

[4] Y. Y. Gu and Y. Zhang, "Photoplethysmographic authentication through fuzzy logic," in Proc. of the IEEE EMBS Asian-Pacific Conf. on Biomedical Engineering, 2003, pp. 136-137.

[5] I. Odinaka, P.-H. Lai, A. Kaplan, J. O'Sullivan, E. Sirevaag, and J. Rohrbaugh, "ECG biometric recognition: A comparative analysis," IEEE Trans. on Information Forensics and Security, vol. 7, pp. 18121824,2012

[6] J. M. Irvine and S. A. Israel, "A sequential procedure for individual identity verification using ecg," EURASIP Journal on Advances in Signal Processing, vol. 2009, pp. 3:1-3:13, January 2009.

[7] S. A. Israel, J. M. Irvine, A. Cheng, M. D. Wiederhold, and B. K Wiederhold, "ECG to identify individuals," Pattern Recognition, vol. 38, pp. 133-142, 2005.

[8] R. Donida Labati, R. Sassi, and F. Scotti, "ECG biometric recognition: Permanence analysis of QRS signals for 24 hours continuous authentication," in Proc. of the IEEE International Workshop on Information Forensics and Security, November 2013, pp. 31-36.

[9] F. Roli, L. Didaci, and G. Marcialis, "Adaptive biometric systems that can improve with use," in Advances in Biometrics, N. Ratha and V. Govindaraju, Eds. Springer London, 2008, pp. 447-471.

[10] U. Uludag, A. Ross, and A. K. Jain, "Biometric template selection and update: a case study in fingerprints," Pattern Recognition, vol. 37, pp. 1533-1542, 2004.

[11] C. Ye, M. Coimbra, and B. Kumar, "Investigation of human identification using two-lead electrocardiogram (ECG) signals," in Proc. of the 2010 Fourth IEEE Int. Conf. on Biometrics: Theory Applications and Systems, 2010, pp. 1-8.

[12] F. Poree, J. Y. Bansard, G. Kervio, and G. Carrault, "Stability analysis of the 12-lead ECG morphology in different physiological conditions of interest for biometric applications," in Computers in Cardiology, 2009, pp. 285-288.

[13] Z. Zhao, L. Yang, D. Chen, and Y. Luo, "A human ECG identification system based on ensemble empirical mode decomposition," Sensors, vol. 13, pp. 6832-6864, 2013.

[14] I. Khalil and F. Sufi, "Legendre polynomials based biometric authentication using QRS complex of ECG," in Proc. of the 2008 Int. Conf. on Intelligent Sensors, Sensor Networks and Information Processing, 2008, pp. 297-302.

[15] K. Sidek, I. Khalil, and M. Smolen, "ECG biometric recognition in different physiological conditions using robust normalized QRS complexes," in Computing in Cardiology, 2012, pp. 97-100.

[16] V. Mai, I. Khalil, and C. Meli, "ECG biometric using multilayer perceptron and radial basis function neural networks," in Proc. of the 2011 Annual Int. Conf. of the IEEE Engineering in Medicine and Biology Society, 2011, pp. 2745-2748.

[17] L. S. Hou, K. Subari, and S. Syahril, "QRS-complex of ECG-based biometrics in a two-level classifier," in Proc. of the 2011 IEEE Region 10 Conf-(TENCON), 2011, pp. 1159-1163.

[18] M. Guennoun, N. Abbad, J. Talom, M. Rahman, and K. El-Khatib, "Continuous authentication by electrocardiogram data," in Proc. of the IEEE Toronto Int. Conf. on Science and Technology for Humanity, 2009, pp. $40-42$

[19] R. Matta, J. Lau, F. Agrafioti, and D. Hatzinakos, "Real-time continuous identification system using ECG signals," in Proc. of the 24th Canadian Conference on Electrical and Computer Engineering, 2011, pp. 13131316.

[20] F. Agrafioti, F. M. Bui, and D. Hatzinakos, "Secure telemedicine: Biometrics for remote and continuous patient verification," Journal Computer Networks and Communications, vol. 2012, 2012.
[21] I. Traore and A. Ahmed, Continuous Authentication Using Biometrics: Data, Models, and Metrics. IGI Global, 2011.

[22] K. Niinuma and A. K. Jain, "Continuous user authentication using temporal information," in Proc. SPIE, Biometric Technology for Human Identification, vol. 7667, 2010.

[23] K. Niinuma, U. Park, and A. Jain, "Soft biometric traits for continuous user authentication," IEEE Trans. on Information Forensics and Security, vol. 5, pp. 771-780, 2010.

[24] T. Sim, S. Zhang, R. Janakiraman, and S. Kumar, "Continuous verification using multimodal biometrics," IEEE Trans. on Pattern Analysis and Machine Intelligence, vol. 29, pp. 687-700, 2007.

[25] A. Azzini, S. Marrara, R. Sassi, and F. Scotti, "A fuzzy approach to multimodal biometric continuous authentication," Fuzzy Optimization and Decision Making, vol. 7, pp. 243-256, September 2008.

[26] A. Rattani, B. Freni, G. Marcialis, and F. Roli, "Template update methods in adaptive biometric systems: A critical review," in Advances in Biometrics, ser. Lecture Notes in Computer Science, M. Tistarelli and M. Nixon, Eds. Springer Berlin Heidelberg, 2009, vol. 5558, pp. $847-856$.

[27] A. Rattani, G. L. Marcialis, and F. Roli, "Biometric system adaptation by self-update and graph-based techniques," Journal of Visual Languages and Computing, vol. 24, pp. 1-9, 2012.

[28] M.-F. Balcan, A. Blum, P. P. Choi, J. D. Lafferty, B. Pantano, M. R. Rwebangira, and X. Zhu, "Person identification in webcam images: An application of semi-supervised learning," in Proc. of the ICML2005 Workshop on Learning with Partially Classified Training Data, 2005.

[29] F. Roli and G. Marcialis, "Semi-supervised pca-based face recognition using self-training," in Structural, Syntactic, and Statistical Pattern Recognition, ser. Lecture Notes in Computer Science, D.-Y. Yeung, J. Kwok, A. Fred, F. Roli, and D. de Ridder, Eds. Springer Berlin Heidelberg, 2006, vol. 4109, pp. 560-568.

[30] X. Jiang and W. Ser, "Online fingerprint template improvement," IEEE Trans. on Pattern Analysis and Machine Intelligence, vol. 24, pp. 11211126, August 2002.

[31] C. Ryu, H. Kim, and A. K. Jain, "Template adaptation based fingerprint verification," in Proc. of the 18th Int.Conf. on Pattern Recognition, 2006, pp. 582-585.

[32] X. Liu, T. Chen, and S. M. Thornton, "Eigenspace updating for nonstationary process and its application to face recognition." Pattern Recognition, vol. 36, pp. 1945-1959, 2003.

[33] K. Okada and C. Von der Malsburg, "Automatic video indexing with incremental gallery creation: integration of recognition and knowledge acquisition," in Proc. of the 3rd Int. Conf. on Knowledge-Based Intelligent Information Engineering Systems, December 1999, pp. 431-434.

[34] J. Weng and W.-S. Hwang, "Toward automation of learning: the state self-organization problem for a face recognizer," in Proc. of the 3rd Int. Conf. on Automatic Face and Gesture Recognition, April 1998, pp. 384-389.

[35] R. Sukthankar and R. Stockton, "Argus: the digital doorman," IEEE Intelligent Systems, vol. 16, pp. 14-19, March 2001.

[36] G. Nagy, "Visual pattern recognition in the years ahead," in Proc. of the 17th Int. Conf. on Pattern Recognition, vol. 4, August 2004, pp. 7-10.

[37] G. Nagy, "Interactive, mobile, distributed pattern recognition," in Image Analysis and Processing, ser. Lecture Notes in Computer Science, F. Roli and S. Vitulano, Eds. Springer Berlin Heidelberg, 2005, vol. 3617, pp. $37-49$.

[38] B. Freni, G. Marcialis, and F. Roli, "Online and offline fingerprint template update using minutiae: An experimental comparison," in Articulated Motion and Deformable Objects, F. Perales and R. Fisher, Eds. Springer Berlin Heidelberg, 2008, vol. 5098, pp. 441-448.

[39] University of Rocher Medical Center, Telemetric and Holter ECG Warehouse, "E-hol-03-0202-003," http://thew-project.org/Database/E-HOL03-0202-003.html. 